Check for updates

Cite this: RSC Adv., 2019, 9, 25880

\title{
A renewable tung oil-derived nitrile rubber and its potential use in epoxy-toughening modifiers $\dagger$
}

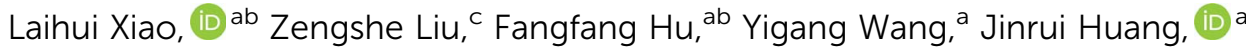 \\ Jie Chen ${ }^{a}$ and Xiaoan Nie ${ }^{* a}$
}

In this study, a modifier (CTMA) prepared by emulsion copolymerization of tung oil fatty acid, methyl esters of tung oil fatty acid and acrylonitrile was used to toughen epoxy resins. The structural characterization of the copolymer was carried out by Fourier transform infrared spectroscopy, ${ }^{1} \mathrm{H}$ NMR spectroscopy and hightemperature gel permeation chromatography. Mechanical testing, thermal characterization and scanning electron microscopy were conducted to investigate the properties of epoxy resin modified by the copolymer and further reveal its toughening mechanism. The results indicated that the newly synthesized copolymer effectively toughened the epoxy resin because the elongation-at-break was increased to $89.48 \%$, the maximum toughness calculated by work before break was nearly 4.6 times that of the neat epoxy resin, and apparent shear yields and plastic deformations were observed in the morphology of the fractured surfaces. CTMA, which acts as a flexible cross-linker in the epoxy thermoset, may decrease the cross-linking density.

Received 13th March 2019

Accepted 17th June 2019

DOI: 10.1039/c9ra01918a

rsc.li/rsc-advances

processing conditions. Therefore, a curing system toughened by modified resins will not always induce phase separation. ${ }^{6-9}$

Among the rubbers used as modifiers, the carboxylterminated copolymer of acrylonitrile and butadiene (CTBN) has been extensively used. The chemical reaction between carboxyl and epoxy groups is able to improve the compatibility between CTBN and epoxy resin, and the long flexible chains may toughen epoxy matrices by developing stress concentrations under stress. ${ }^{10-12}$ Laura et al. have studied CTBN-modified epoxy resin by laser confocal microscopy characterization and found that the fracture toughness increases, whereas the glass transition temperature and the tensile modulus decrease as the size and phase volume of CTBN increase ${ }^{13}$ Huang et al. have prepared a flexible and transparent epoxy resin with tunable mechanical properties by the reaction between hydrazine hydrate and the epoxy functional group diglycidyl ether of bisphenol A (DGEBA). The curing systems incorporated with the modified resin demonstrated excellent flexibility; however, no phase separation was observed. ${ }^{14}$ Day et al. have toughened epoxy resin with a series of multilayer core-shell particles. Compared to acrylic toughening particles (ATP) and CTBN under the same conditions, the epoxy resin modified by these multilayer core-shell particles improved the toughness, which led to a significant decrease in Young's modulus. ${ }^{15}$ Fei et al. used hyperbranched polymers to toughen the epoxy resin that were synthesized from the diglycidyl ether of bisphenol A, succinic acid (SA) and 1,3,5-benzenetricarboxylic acid (BA). After being cured by methylhexahydrophthalic anhydride (MeHHPA), the modified epoxy thermosets exhibited better toughness as the elongation-at-break of $5.25 \%$ could be achieved as 
compared to $3 \%$ of the neat epoxy thermoset; in addition, the glass transition temperatures of the modified epoxy thermosets increased because of their hyperbranched structure. ${ }^{8}$

However, due to the growing concerns over sustainability and environmental friendliness, the use of renewable materials is necessary; therefore, traditional methods of toughening the epoxy resin by petroleum-based modified resins are being gradually replaced by those involving the use of renewable materials. Thus, the synthesis of modifiers partly or completely from renewable feedstock is urgent.

Vegetable oils (VOs) are excellent alternatives due to their abundance, low price and biodegradability. ${ }^{16,17}$ Many studies have been devoted towards the toughening of epoxy resin by epoxidized vegetable oils. ${ }^{18,19}$ These epoxidized vegetable oils are incorporated into epoxy thermosets as a flexible crosslinker, which toughen the epoxy resin by decreasing the crosslinking density. Ratna et al. toughened DGEBA by epoxidized soybean oil using an ambient-temperature hardener. When the addition amount of the modifier was increased from 5\% to $30 \%$, the maximum elongation-at-break obtained was $7.5 \%$ (which was $3.2 \%$ for the sample without the modifier); this led to a significant improvement in toughness $;^{20}$ however, it still could not satisfy the requirement for application in certain special occasions.

Moreover, the conjugated structure in tung oil obtained via the Diels-Alder reaction rather than epoxidization has received significant research interest. By the Diels-Alder reaction, a number of tung oil-derived toughened epoxy resins have been produced. ${ }^{21-24}$ As is well-known, the products obtained via the Diels-Alder reaction contain a ring structure, which may reduce the toughening effect. Hence, to avoid the formation of a ring structure, an alternative strategy is to synthesize the tung oilbased toughener via radical polymerization; however, only few studies have been reported on the synthesis of an epoxy resin toughener by the radical polymerization of tung oil or tung oilderives.

In this study, a renewable toughener was synthesized by the emulsion polymerization of tung oil fatty acid (TOFA), methyl esters of tung oil fatty acid (METOFA) and acrylonitrile. The long flexible aliphatic chains of tung oil-derives were able to toughen the rigid epoxy matrix, and the carboxyl groups of TOFA improved the compatibility of blends. Then, to study the effect of the addition content of the synthesized resin on the properties of the modified epoxy resin, the synthesized resin in different contents was incorporated into the epoxy matrix by a pre-reaction between the carboxyl groups and epoxy groups. After curing, the mechanical properties and thermal characterizations were investigated by mechanical testing, differential scanning calorimetry (DSC), dynamic mechanical analysis (DMA) and thermogravimetric analysis (TGA). In addition, the fracture surfaces were observed by a scanning electron microscope (SEM). The results of this study show that the synthesized copolymer resin can effectively toughen the epoxy resin.

\section{Experimental}

\section{Material}

Tung oil was obtained from the Institute of Chemical Industry of Forestry Products. Diglycidyl ether of bisphenol A (epoxide value: $0.48-0.54$ equiv. $\left.(100 \mathrm{~g})^{-1}\right)$, Baling Petrochemical Branch Company, PetroChina Co., Ltd, polyetherimide (D230, amine value: 8.1-8.7 mequiv. $\mathrm{g}^{-1}$, Huntsman Chemical Co., Ltd), triphenylphosphine (99.5\%, Shanghai Lingfeng Chemical Reagent Co., Ltd), acrylonitrile (98\%, Sinopec Shanghai Petrochemical Co., Ltd), potassium oleate (98\%, Shanghai Macklin Biochemical Co., Ltd), ammonium persulphate (98\%, Shanghai Lingfeng Chemical Reagent Co., Ltd), sodium chloride (Xilong Scientific Co., Ltd), sodium hydroxide (Xilong Scientific Co., Ltd), potassium hydroxide (Xilong Scientific Co., Ltd), methanol (99.5\%, Nanjing Chemical Reagent Co., Ltd), hydrochloric acid (36.0$38.0 \%$, Nanjing Chemical Reagent Co., Ltd) and epoxidized soybean oil (epoxide value: 0.38 equiv. (100 g) $)^{-1}$, Shanghai Aladdin Chemical Reagent Co., Ltd) were used as received.

\section{Synthesis}

Tung oil fatty acids (TOFA). Tung oil (180 g) and $\mathrm{NaOH}$ solution $(100 \mathrm{~mL})$ prepared by dissolving $24 \mathrm{~g}$ of sodium hydroxide in $100 \mathrm{~mL}$ water/ethanol $(1: 1 \mathrm{v} / \mathrm{v})$ were taken in a flask, followed by refluxing for $3 \mathrm{~h}$ at $75^{\circ} \mathrm{C}$. After decomposing the soaps by hydrochloric acid, the water layer was removed. The crude product was rinsed with water, and the trace amount of water was removed by a rotary evaporator under vacuum at $70{ }^{\circ} \mathrm{C}$. Then, $161 \mathrm{~g}$ of a light brown product was obtained, whose acid value was $200.3 \mathrm{mg} \mathrm{g}^{-1}$ (theoretical: $201.8 \mathrm{mg} \mathrm{g}^{-1}$ ).

Methyl esters of tung oil fatty acids (METOFA). Tung oil (200 $\mathrm{g})$, methanol (54 g) and potassium hydroxide ( $3 \mathrm{~g}$ ) were charged into a three-necked flask fitted with a mechanical stirrer and refluxed at $70{ }^{\circ} \mathrm{C}$ for $1.5 \mathrm{~h}$. After the excess methanol was removed using a rotary evaporator under vacuum, the product was easily separated from the reaction mixture. The purification methods are the same as those used for TOFA, and $187 \mathrm{~g}$ of a yellow product was obtained.

Copolymer of tung oil fatty acids, methyl esters of tung oil fatty acids and acrylonitrile (CTMA). TOFA (15 g), METOFA (15 $\mathrm{g})$, acrylonitrile $(20 \mathrm{~g})$, potassium oleate $(0.5 \mathrm{~g})$ and water $(100 \mathrm{~g})$ were charged into a round-bottomed flask fitted with a magnetic stirrer and a condenser. Under a nitrogen atmosphere, the reaction system was heated to $70{ }^{\circ} \mathrm{C}$, and ammonium persulphate $(0.4 \mathrm{~g}$ ammonium persulphate was dissolved in $20 \mathrm{~g}$ water beforehand) was added to the mixture. After stirring at $70{ }^{\circ} \mathrm{C}$ for $10 \mathrm{~h}$, the product was agglomerated by adding $\mathrm{NaCl}(10 \mathrm{~g})$. The crude product was purified by acetone and water and dried under vacuum at $75^{\circ} \mathrm{C}$. Then, $36.8 \mathrm{~g}$ of a viscous product was obtained.

The preparation method is shown in Fig. 1.

\section{Preparation of the CTMA-toughened epoxy resin (E-CTMA) and curing procedure}

Epoxy resin and CTMA (in the mass ratios of $100: 25,100: 35$, and $100: 45)$ were charged into a flask. After catalysis by 


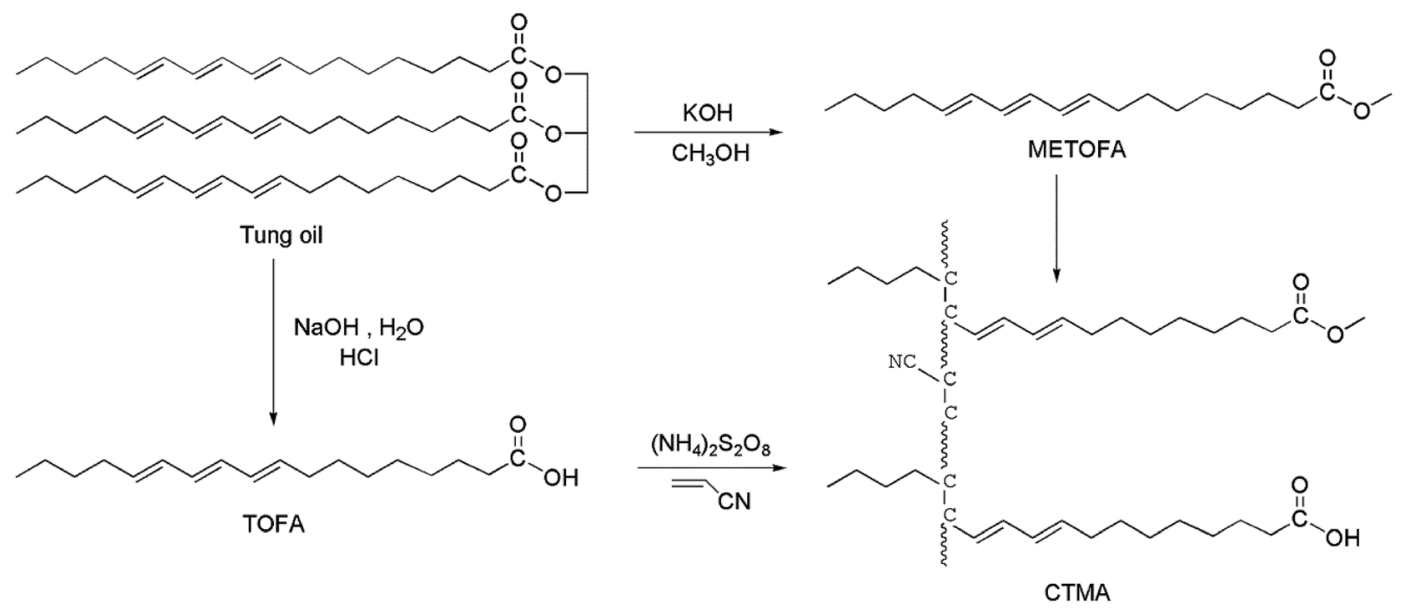

Fig. 1 Synthesis routes of CTMA.

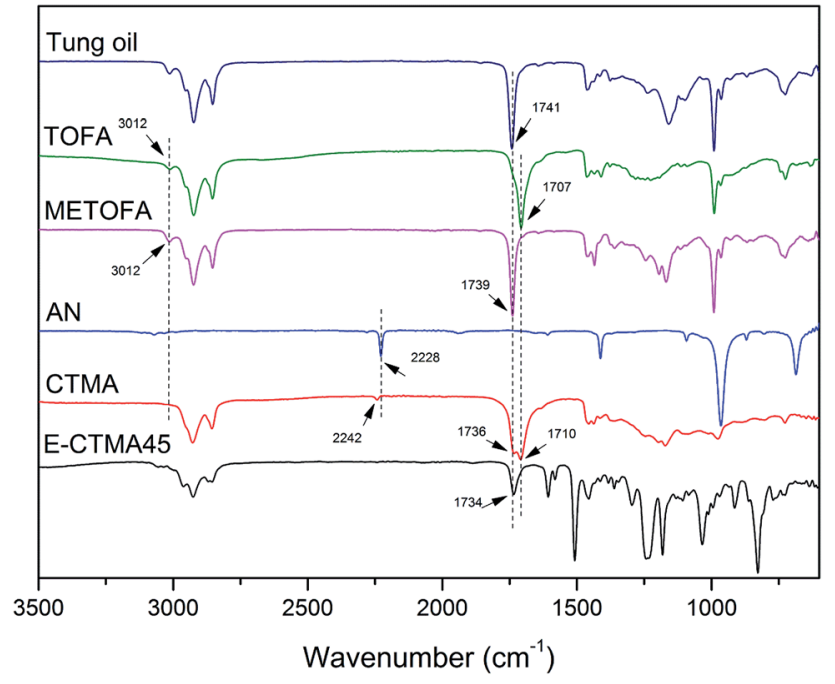

Fig. 2 FTIR spectra of CTMA and materials.

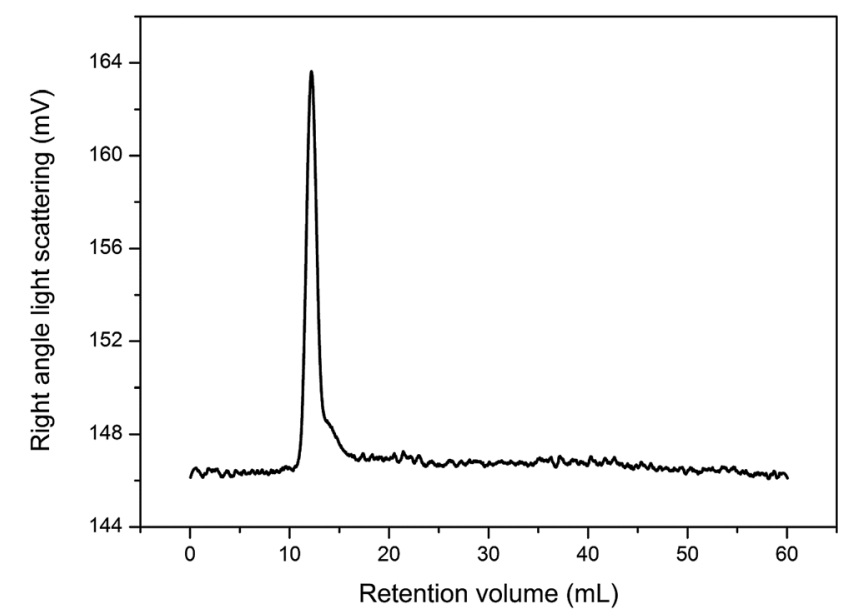

Fig. 3 GPC spectrum of CTMA. triphenylphosphine ( $0.2 \mathrm{wt} \%$ of epoxy resin), the mixture was stirred at $130{ }^{\circ} \mathrm{C}$ for $3 \mathrm{~h}$. After the product was cooled down to room temperature, polyetherimide was added (epoxy and curing agent were in the $1: 1$ equivalent ratio). The mixture was then charged into a stainless mold preheated at $80{ }^{\circ} \mathrm{C}$. Curing was performed at $40{ }^{\circ} \mathrm{C}$ for $24 \mathrm{~h}$ and at $80{ }^{\circ} \mathrm{C}$ for $3 \mathrm{~h}$. These samples were then coded as E-CTMA25, E-CTMA35 and ECTMA45. Epoxidized soybean oil (ESO) was blended with epoxy resin in the mass ratio of $100: 45$ at room temperature, and the sample was coded as E-ESO45, which was then used for comparison purposes. Neat epoxy resin (EP) was also prepared in the same way as a control group.

\section{Characterization}

Structural characterization. Fourier transform infrared (FTIR) analysis was performed using the Thermo Scientific Nicolet iS10 spectrometer in the wavenumber range of 600$4000 \mathrm{~cm}^{-1}$. The ${ }^{1} \mathrm{H}$ NMR spectra of tung oil-derives and CTMA were obtained by the Bruker $400 \mathrm{MHz}$ spectrometer at room temperature using deuterated chloroform $\left(\mathrm{CDCl}_{3}\right)$ as a solvent. The number-average molecular weight $\left(M_{\mathrm{n}}\right)$ and dispersity $(D)$ were determined using the Viscotek model 350 hightemperature gel permeation chromatograph (HTGPC) at $60{ }^{\circ} \mathrm{C}$ with $N, N$-dimethylformamide (DMF) as the solvent. The flow

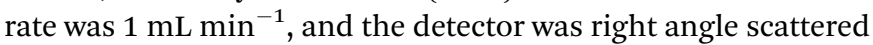
light, whose temperature was $60{ }^{\circ} \mathrm{C}$.

Thermal characterization. Differential scanning calorimetry (DSC) was conducted using a Diamond DSC (PE) instrument. The samples $5-10 \mathrm{mg}$ in weight were placed in aluminum pans under a nitrogen atmosphere. After being heated from $-50{ }^{\circ} \mathrm{C}$ to $180{ }^{\circ} \mathrm{C}$ at $10{ }^{\circ} \mathrm{C} \mathrm{min}{ }^{-1}$ and maintained isothermally for $5 \mathrm{~min}$ to eliminate the thermal history, the samples E-CTMAs and neat epoxy resin were cooled down to $-50{ }^{\circ} \mathrm{C}$ and reheated to $180{ }^{\circ} \mathrm{C}$ under the same conditions. The operations for the sample CTMA were the same as those used for E-CTMAs, except that the temperature range was from $-70{ }^{\circ} \mathrm{C}$ to $80{ }^{\circ} \mathrm{C}$. Moreover, the curing behaviour was studied by DSC, and all samples were scanned from $0{ }^{\circ} \mathrm{C}$ to 

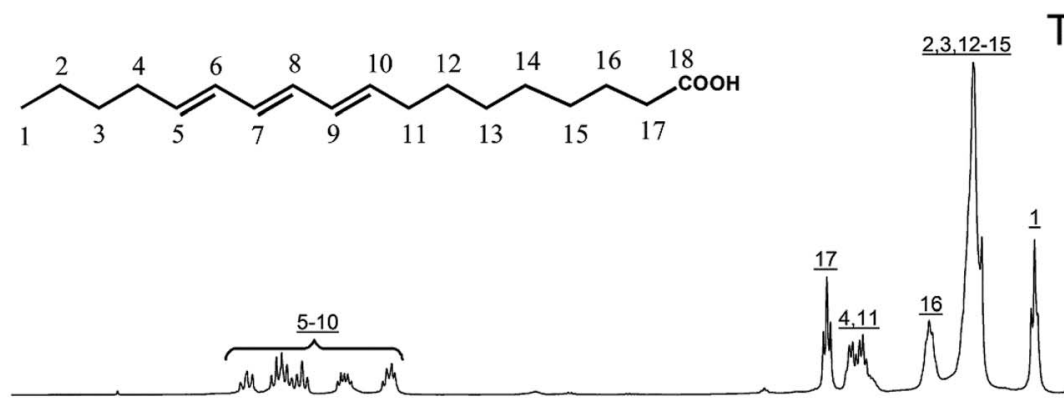

\section{TOFA}
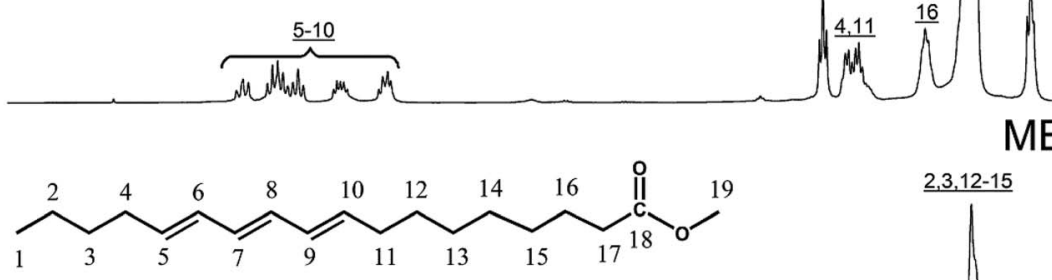

METOFA
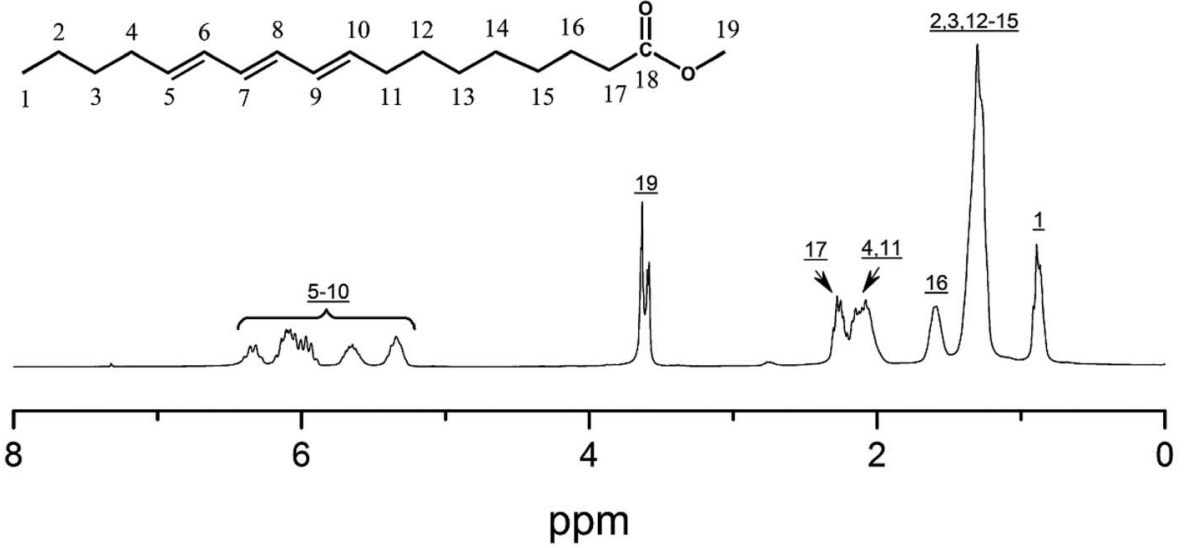

Fig. $4{ }^{1} \mathrm{H}$ NMR spectra of TOFA and METOFA.

$250{ }^{\circ} \mathrm{C}$ at $10{ }^{\circ} \mathrm{C} \mathrm{min}^{-1}$. Dynamic mechanical analysis (DMA) of the samples was performed by DMA Q800 (TA Instruments) in the dual cantilever mode with the oscillating frequency of $1 \mathrm{~Hz}$. The temperature range from $-50{ }^{\circ} \mathrm{C}$ to $150{ }^{\circ} \mathrm{C}$ at
$3{ }^{\circ} \mathrm{C} \min ^{-1}$ was used. Thermogravimetric analysis (TGA) was performed using the TG209F1 TGA (Netzsch) instrument. Each sample was scanned from $30^{\circ} \mathrm{C}$ to $800{ }^{\circ} \mathrm{C}$ at $20^{\circ} \mathrm{C} \mathrm{min}^{-1}$ under a nitrogen atmosphere.

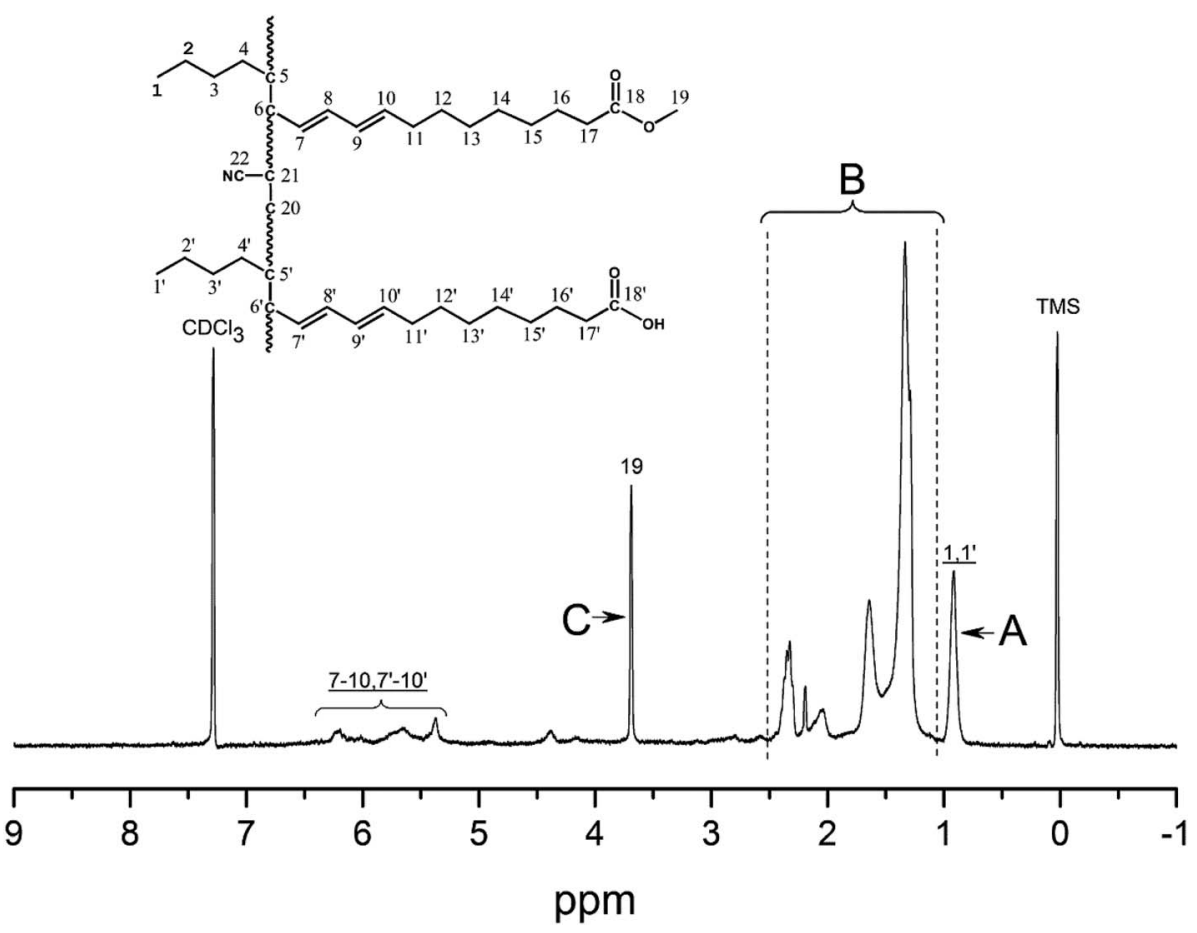

Fig. $5{ }^{1} \mathrm{H}$ NMR spectrum of CTMA. 
Table 1 Calculation results obtained by the ${ }^{1} \mathrm{H}$ NMR spectra

\begin{tabular}{llll}
\hline Part & $A$ & $B$ & $C$ \\
\hline Peak area (relative to $C$ ) & 1.75 & 14.28 & 1 \\
Monomer & AN & METOFA & TOFA \\
Mole fraction (\%) & 45.26 & 31.28 & 24.06
\end{tabular}

Scanning electron microscopy characterization. Surface morphology of the samples was determined using the $3400 \mathrm{NI}$ scanning electron microscope (Hitachi) at the acceleration voltage of $15 \mathrm{kV}$. All samples were fractured after being placed in liquid nitrogen and coated with gold before examination.

Mechanical properties. Tensile tests were conducted using the Instron 4201 machine equipped with a $30 \mathrm{kN}$ electronic load cell according to the Chinese standard GB13022-91. Moreover, five replicates for each sample were tested at the crosshead speed of $5 \mathrm{~mm} \min ^{-1}$ at $25{ }^{\circ} \mathrm{C}$.

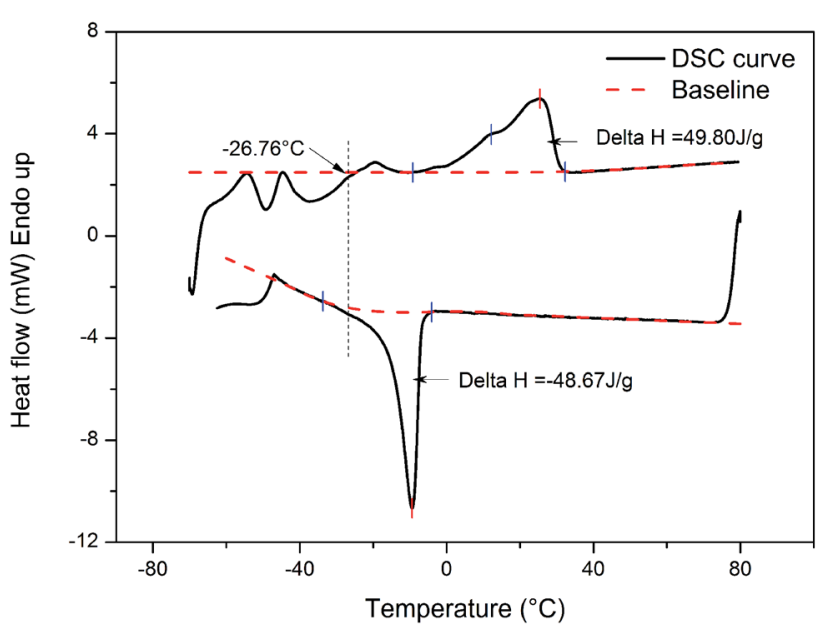

(a)

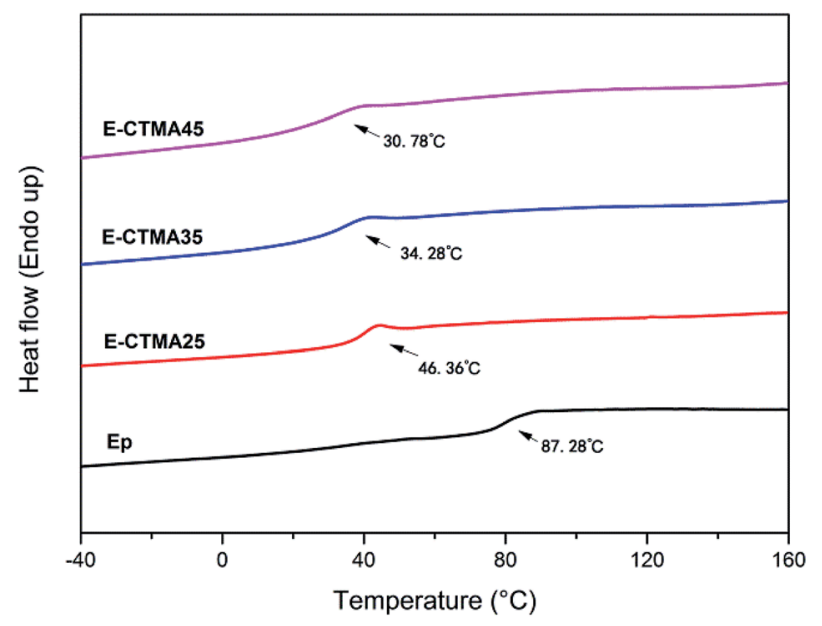

(b)

Fig. 6 DSC curves of CTMA (a) and cured thermosets (b).

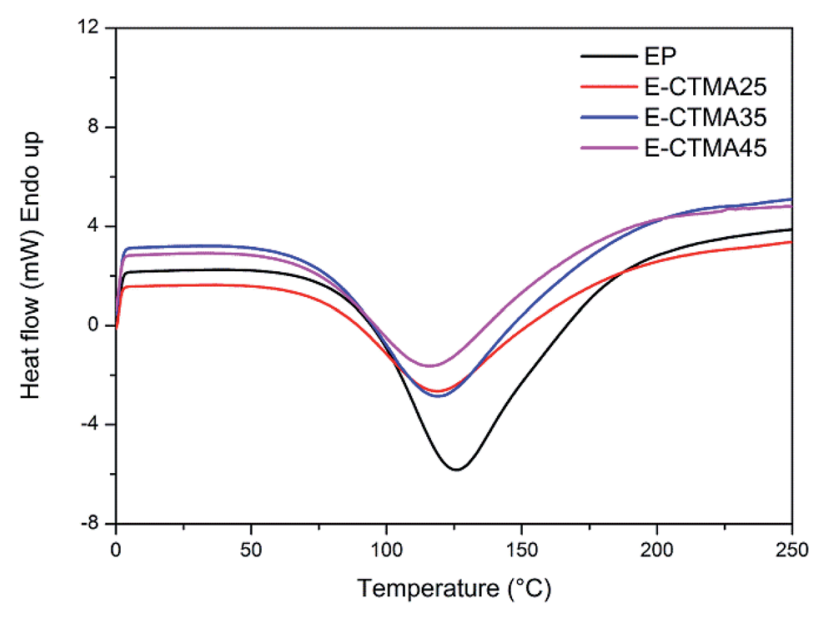

(a)

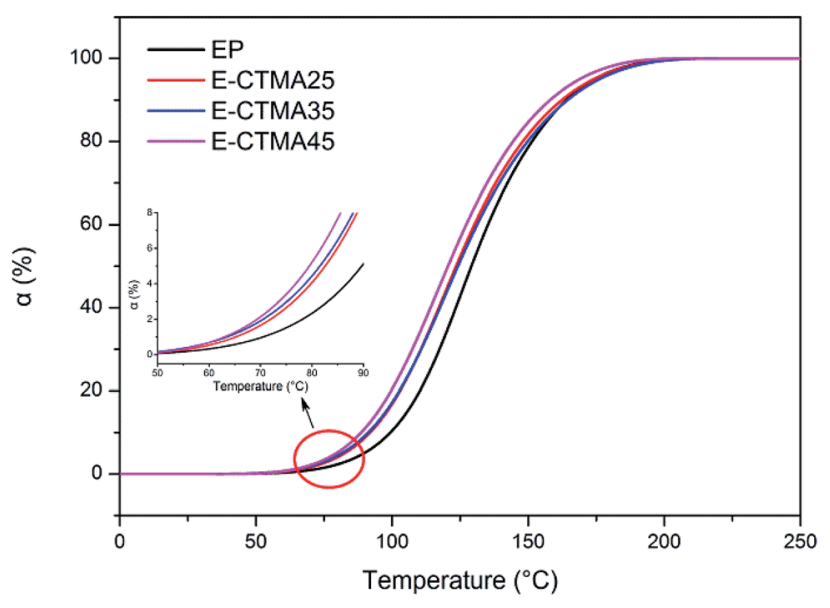

(b)

Fig. 7 DSC curves of the curing process: heat flow against temperature (a) and conversion against temperature (b).

\section{Results and discussion}

\section{Structure determinations}

As shown in Fig. 2, functional group conversion can be detected by comparing the infrared spectra. In the CTMA spectrum, a weaker peak of $=\mathrm{C}-\mathrm{H}$ appeared at $3012 \mathrm{~cm}^{-1}$ as compared to that of tung oil-derives, which indicated the polymerization of

Table 2 Curing parameters and viscosity of samples

\begin{tabular}{lllll}
\hline Sample & $T_{\mathrm{o}}{ }^{a}\left({ }^{\circ} \mathrm{C}\right)$ & $T_{\mathrm{p}}{ }^{b}\left({ }^{\circ} \mathrm{C}\right)$ & $\Delta H\left(\mathrm{~J} \mathrm{~g}^{-1}\right)$ & Viscosity $^{c}(\mathrm{kPa} \mathrm{s})$ \\
\hline EP & 89.17 & 126.18 & 379.65 & 12.36 \\
E-CTMA25 & 78.37 & 119.43 & 297.89 & 19.16 \\
E-CTMA35 & 77.89 & 119.25 & 292.83 & 27.08 \\
E-CTMA45 & 74.43 & 116.78 & 244.34 & 51.22
\end{tabular}

${ }^{a} T_{\mathrm{o}}$ is the initial curing temperature. ${ }^{b} T_{\mathrm{p}}$ is the temperature at which the curing rate is maximum. ${ }^{c}$ Viscosity is measured at room temperature. 
the double bond. Furthermore, the peaks of $-\mathrm{COOCH}_{3}$ at $1739 \mathrm{~cm}^{-1},-\mathrm{COOH}$ at $1707 \mathrm{~cm}^{-1}$ and $-\mathrm{CN}$ at $2228 \mathrm{~cm}^{-1}$ were observed in the CTMA spectrum, indicating that CTMA contained these three monomer units. Due to the reaction between the carboxyl groups and the epoxy groups, the peak of $-\mathrm{COOH}$ at $1707 \mathrm{~cm}^{-1}$ disappeared in the spectrum of E-CTMA45.

Fig. 3 shows the GPC spectrum of CTMA. Only one peak was observed in the spectrum, which confirmed that the main product was the copolymer of the three monomer units. The number-average molecular weight is about $28740 \mathrm{~g} \mathrm{~mol}^{-1}$, and the $D$ value is 5.43. The high $D$ value may be attributed to the different monomer activities of acrylonitrile and tung oilderives. Furthermore, the simultaneous addition of monomers contributed to high $D$ value.

Fig. 4 and 5 show the ${ }^{1} \mathrm{H}$ NMR spectra of tung oil-derives and CTMA, which have been used to determine the copolymer composition. In the ${ }^{1} \mathrm{H}$ NMR spectrum of CTMA, the proton resonances of $-\mathrm{COOCH}_{3}(3.69 \mathrm{ppm})$ in METOFA and $-\mathrm{CH}_{3}(0.91$ ppm) in TOFA and METOFA are well separated, whereas those

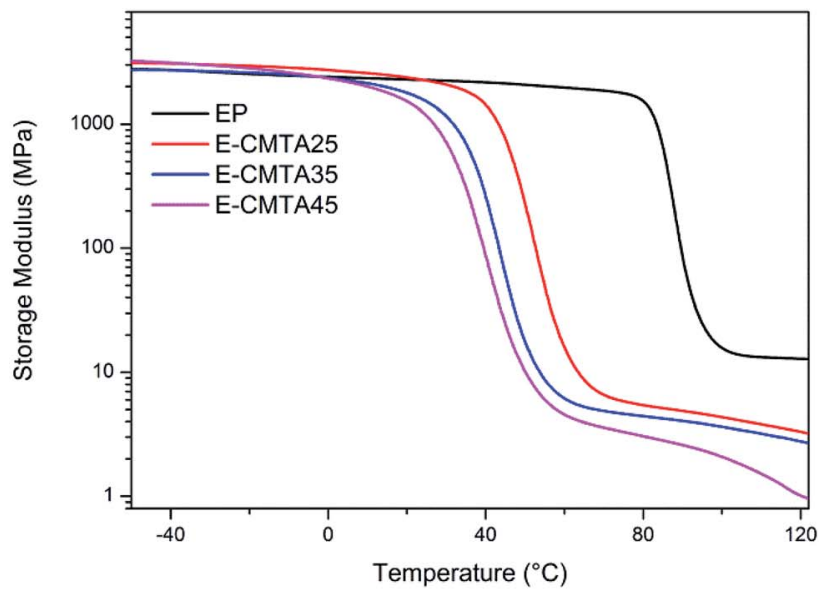

(a)

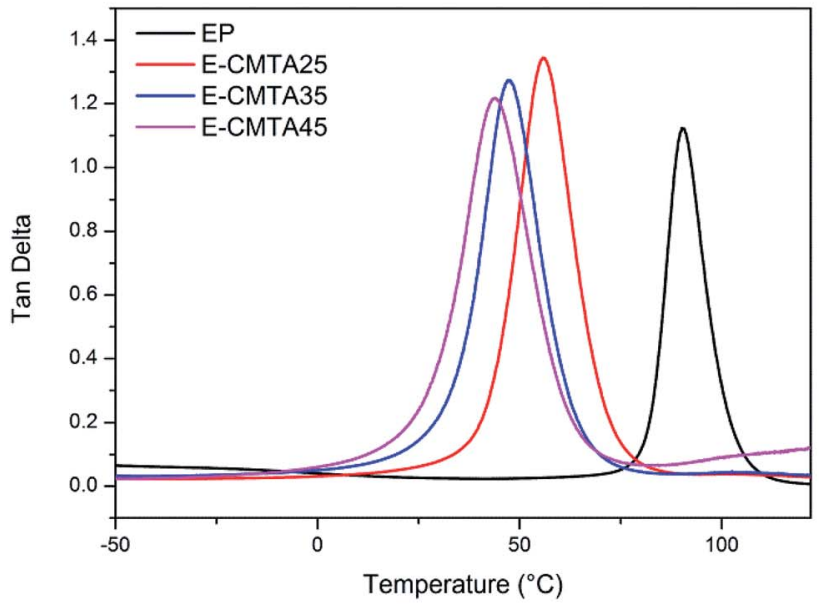

(b) for methylene protons and methine protons (1-2.5 ppm) are overlapped. Hence, their relative peak areas can be used to calculate the mole fraction of the monomer by the following formulas,

$$
\begin{gathered}
F(\mathrm{AN})=\frac{\left(B-\frac{A}{3} \times 22\right) / 3}{\frac{A}{3}+\left(B-\frac{A}{3} \times 22\right) / 3} \\
F(\text { METOFA })=\frac{C / 3}{\frac{A}{3}+\left(B-\frac{A}{3} \times 22\right) / 3} \\
F(\text { TOFA })=\frac{(A-C) / 3}{\frac{A}{3}+\left(B-\frac{A}{3} \times 22\right) / 3}
\end{gathered}
$$

where $A$ and $C$ are the peak areas for the proton resonances of $-\mathrm{CH}_{3}$ and $-\mathrm{COOCH}_{3}$, respectively. $B$ is the peak area for all the methylene proton and methine proton resonances, which include those of the tung oil derive and acrylonitrile. $F$ (AN), $F$ (METOFA) and $F$ (TOFA) correspond to the mole fraction of AN, METOFA and TOFA, respectively. The calculation results are presented in Table 1.

\section{Differential scanning calorimetry}

Fig. 6 shows the results of DSC. In Fig. 6(a), we can observe a melting peak and a crystallization peak, which illustrates the crystallizability of CTMA. Moreover, the approximate enthalpy values confirm the corresponding melt crystallization process. As observed in the CTMA structure shown in Fig. 1, the flexibility of the long aliphatic chain causes the molecules to align; this leads to crystallization. Furthermore, the induction forces induced by the cyano groups were able to stabilize the crystallization. Note that via both the heating curve and the cooling curve, the glass transition temperature of CTMA can be detected, which is around $-26.76^{\circ} \mathrm{C}$.

However, no melting or crystallization peaks were observed in the curves of E-CTMA, and only one glass transition temperature could be detected, which indicated that no phase separation occurred. This was because the epoxy resin incorporated CTMA by the reaction between the carboxyl groups and the epoxy groups; this contributed to the molecular-level compatibility.

As flexible cross-linkers, many long flexible aliphatic chains were incorporated into E-CTMA thermosets. Segment

Table 3 DMA results of the neat epoxy resin and E-CTMA thermosets

\begin{tabular}{lllrl}
\hline Sample code & $T_{\mathrm{g}}\left({ }^{\circ} \mathrm{C}\right)$ & Half-peak width $\left({ }^{\circ} \mathrm{C}\right)$ & $E^{\prime a}$ & \multicolumn{2}{l}{$v_{\mathrm{e}}\left(10^{-3} \mathrm{~mol} \mathrm{~cm}^{-3}\right)$} \\
\hline EP & & & 12.86 & 1.31 \\
E-CTMA25 & 90.15 & 11.12 & 5.10 & 0.57 \\
E-CTMA35 & 47.38 & 16.27 & 4.53 & 0.52 \\
E-CTMA45 & 43.77 & 19.93 & 3.36 & 0.39
\end{tabular}

Fig. 8 Storage modulus (a) and $\tan \delta$ (b) of the neat epoxy resin and E- $\quad{ }^{a}$ Storage modulus at $T_{\mathrm{g}}+30{ }^{\circ} \mathrm{C}$. CTMA thermosets. 
movement in flexible aliphatic chains can be easily induced by increasing the temperature, which will contribute to a lower glass transition temperature; furthermore, the high crosslinking network system will be destroyed, and the less restriction from covalent bonds contributes to segment movement, which will also contribute to a lower glass transition temperature. All these results are in accordance with the decreasing $T_{\mathrm{g}}$ value shown in Fig. 6(b); moreover, further explanation about this point has been provided in the discussion of DMA.

Furthermore, the curing behavior was studied by DSC. In Fig. 7(a), all samples show a similar curing behavior, and only one curing process has been determined. The accurate parameters of the curing process are presented in Table 2. Obviously, the $T_{\mathrm{o}}$ and $T_{\mathrm{p}}$ values of E-CTMAs are significantly lower than that of the neat epoxy resin, and a decreasing tendency is observed as the content of CTMA is increased. Previous studies have demonstrated that the existence of strong hydrogen bonding species, such as acids and alcohols, is able to accelerate the epoxy-amine reaction. ${ }^{25}$ In the blending process, the carboxyl groups in CTMA react with the epoxy groups, and the same amount of hydroxyls are simultaneously produced. Therefore, the curing process is accelerated, which is in agreement with the decreased values of $T_{\mathrm{o}}$ and $T_{\mathrm{p}}$. Furthermore, this positive effect on the curing process is shown in Fig. 7(b) in which it is observed that CTMA enhances the conversion at a specific temperature; however, higher content of CTMA results in lower enthalpy $(\Delta H)$. This is because the addition of CTMA reduces the content of epoxy groups and curing agent; this leads to fewer reactions. Moreover, the incorporation of CTMA increases the viscosity of epoxy resin (Table 2), which is attributed to the much higher molecular weight of CTMA. Furthermore, the pre-reaction between the carboxyl and the epoxy groups may produce hydroxyls, which may form hydrogen bonds with other polar groups and further increase the viscosity. Similar results have been obtained in previous studies, in which rubber and other oil-based polymers have been used to toughen the epoxy resin. ${ }^{12,21,26}$ The increased viscosity may restrict the movement of reaction groups and then reduce the enthalpy.

\section{Dynamic mechanical analysis}

Fig. 8 shows the temperature dependence of storage modulus and $\tan \delta$ of the neat epoxy resin and E-CTMA thermosets. The accurate statistics of thermal properties are presented in Table 3.

$T_{\mathrm{g}}$ decreases with an increase in the CTMA content; this is in accordance with the abovementioned DSC results provided in the DSC section. Furthermore, the explanation of the decreasing $T_{\mathrm{g}}$ can be confirmed by the results of half-peak width (HPW) in $\tan \delta$ curves, which is related to structural uniformity. ${ }^{27}$ The neat epoxy resin has the lowest HPW value, whereas E-CTMA45 has the highest HPW value of E-CTMA. This is because CTMA acts as a flexible crosslinker, which increases the molecular weight between cross-linking points. Furthermore, the random carboxyl distribution of CTMA extends the

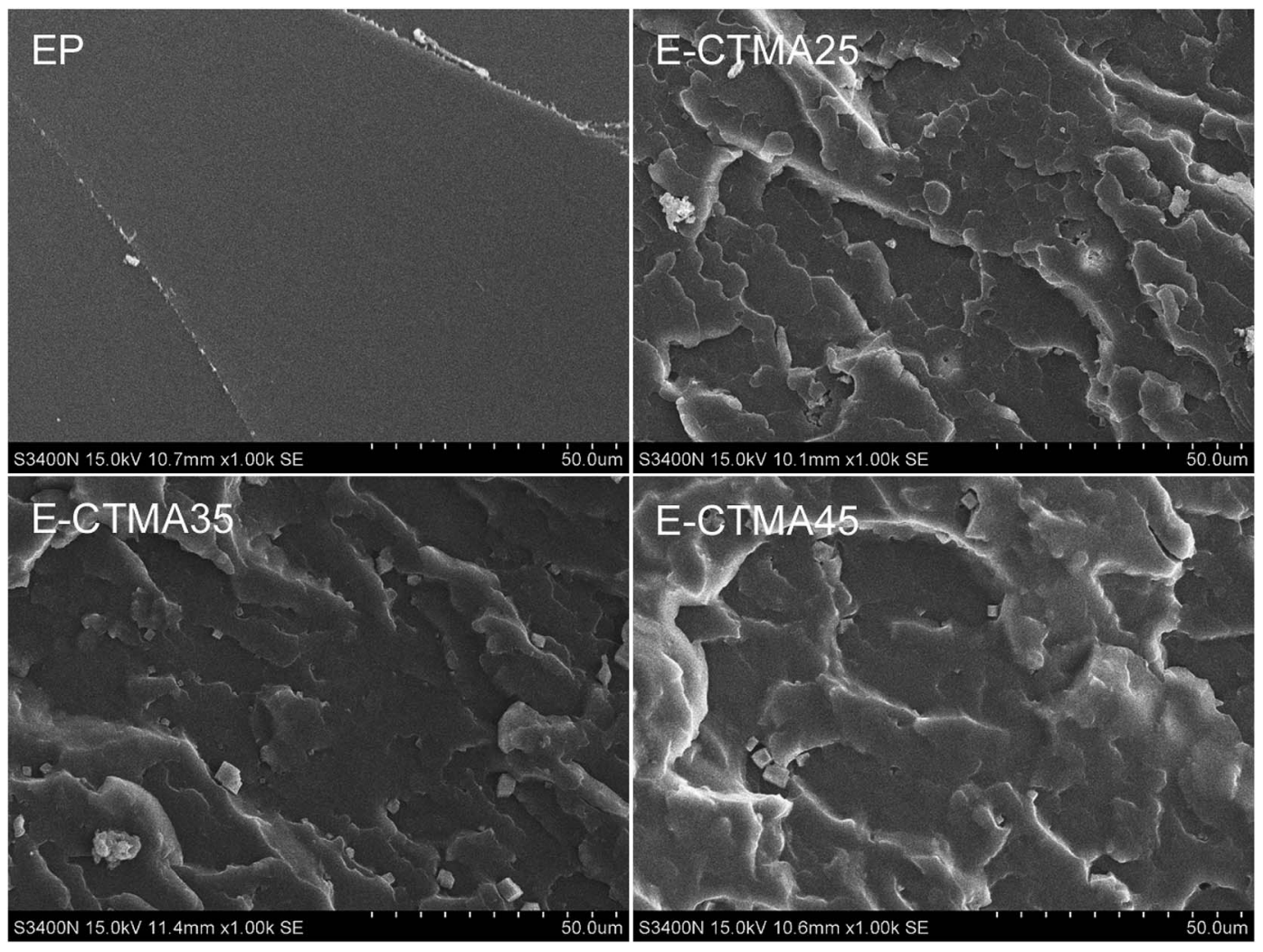

Fig. 9 SEM images of the frozen fracture morphology for the thermosets: neat epoxy, E-CTMA25, E-CTMA35 and E-CTMA45. 
dispersity of molecular weight between cross-linking points. However, every $\tan \delta$ curve of E-CTMAs and neat epoxy resin has only one peak, which illustrates that the uniform network structure is unable to lead to phase separation. ${ }^{28,29}$

Based on the theory of rubber elasticity, the cross-linking density $v_{\mathrm{e}}$ can be calculated from the storage modulus in the rubbery region:

$$
v_{\mathrm{e}}=\frac{E^{\prime}}{3 R T}
$$

where $E^{\prime}$ is the storage modulus at $T_{\mathrm{g}}+30{ }^{\circ} \mathrm{C}, R$ is the gas constant, and $T$ is the absolute temperature at $T_{\mathrm{g}}+30{ }^{\circ} \mathrm{C} .{ }^{\mathbf{1 4 , 2 4}}$

The value of $v_{\mathrm{e}}$ decreases with the increasing content of CTMA; this indicates lower cross-linking density. With fewer cross-linking points, chain segments move more easily; this contributes to a lower glass transition temperature. Thus, the decreasing glass transition temperatures are presented as $T_{\mathrm{g}}$ value in Table 3 .

\section{Morphology of the fractured surfaces}

In the SEM images shown in Fig. 9, we can observe that the fracture surface of neat epoxy samples is very smooth, whereas that of the CTMA-modified epoxy samples is rather rough. No cavity was observed on the fracture surfaces; this indicated that no phase separation occurred. ${ }^{\mathbf{1 4 , 3 0}}$ This result is in line with the results of DSC and DMA. Furthermore, massive shear yields and plastic deformations observed on the E-CTMA fracture surfaces indicate a typical toughness character.

When CTMA was added as a modifier, the carboxyl groups reacted with the epoxy groups; this led to molecular level incorporation instead of phase separation. This phenomenon has also been observed by other researchers. ${ }^{31-34}$ Because of the long aliphatic chains of CTMA, external stresses can be redistributed, and plastic deformations will be induced by the flexible cross-linker. This is the reason why the fracture surfaces become rougher.

\section{Thermogravimetric analysis}

The thermal stability of CTMA and the neat epoxy resin and ECTMA thermosets was examined, as shown in Fig. 10. We can observe that E-CTMAs tend to decompose at lower temperatures, $T_{5 \%}$ and $T_{10 \%}$ presented in Table 4 ; this is attributed to the decomposition of small molecules (carbonyl and ester) in CTMA. Consequently, a subtle peak at $250{ }^{\circ} \mathrm{C}$ appears in the derivative curves with an increase in CTMA content, which has been magnified in the inset of Fig. 10(b). The peak around $395{ }^{\circ} \mathrm{C}$ in the derivative curves demonstrates the main decomposition process, which is considered to be caused by the degradation of the cross-linked epoxy resin networks. ${ }^{35,36}$ Hence, the maximum degradation rate temperatures of E-CTMA and neat epoxy resin thermosets are approximate. Similar results have been reported by other researches..$^{35,37-39}$ Kumar et al. toughened the epoxy resin using ESO in different contents, and the results show that $T_{5 \%}$ decreases from $341{ }^{\circ} \mathrm{C}$ to $283{ }^{\circ} \mathrm{C}$ when the mass ratio between epoxy resin and ESO is $80 / 20 ;^{38}$ however, CTMA leads to a greater weight loss; this may be

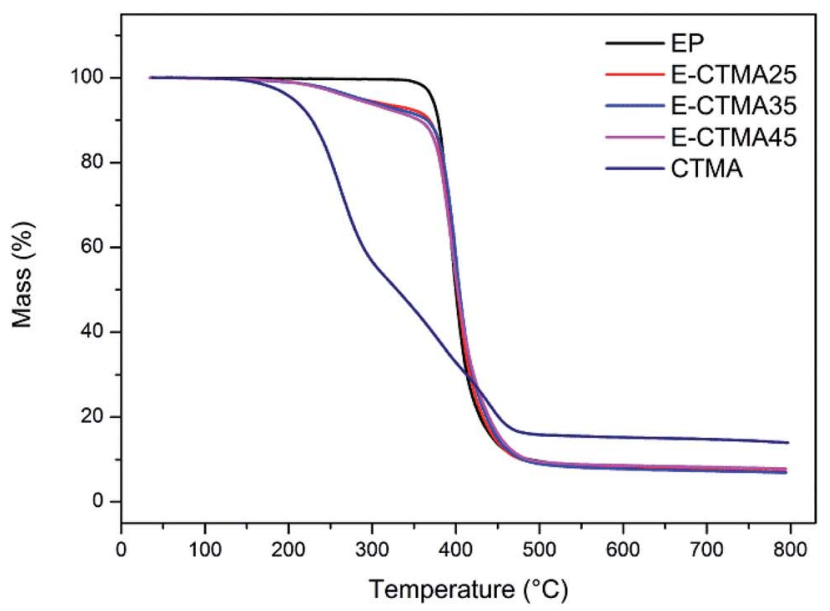

(a)

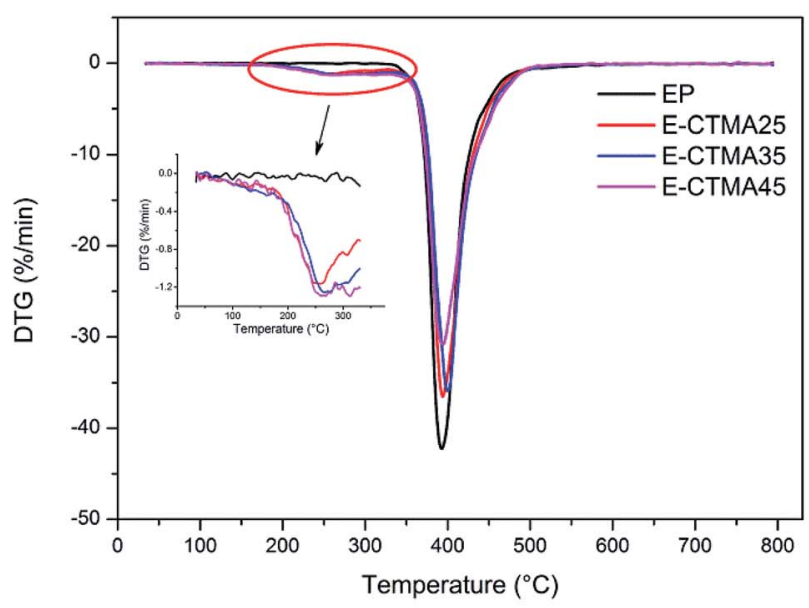

(b)

Fig. 10 Thermogravimetric plots of the curing system: TG curves (a) and DTG curves (b).

explained in terms of the cross-linking density. As is wellknown, higher cross-linking density contributes to higher thermal stability. ${ }^{38}$ The incorporation of CTMA results in a great loss in the cross-linking density, which may account for the loss in thermal stability. However, the epoxide equivalent weight of

Table 4 Thermal properties of the neat epoxy resin and E-CTMA thermosets

\begin{tabular}{llll}
\hline Sample code & $T_{5 \%}{ }^{a}\left({ }^{\circ} \mathrm{C}\right)$ & $T_{10 \%}{ }^{a}\left({ }^{\circ} \mathrm{C}\right)$ & $T_{\mathrm{m}}{ }^{b}\left({ }^{\circ} \mathrm{C}\right)$ \\
\hline EP & 372.06 & 378.92 & 392.7 \\
E-CTMA25 & 286.11 & 368.04 & 394.0 \\
E-CTMA35 & 285.91 & 365.29 & 400.2 \\
E-CTMA45 & 279.49 & 355.32 & 393.8 \\
CTMA & 204.94 & 227.84 & 263.9
\end{tabular}

${ }^{a} T_{5 \%}$ and $T_{10 \%}$ are the temperatures of $5 \%$ and $10 \%$ weight loss, respectively. ${ }^{b} T_{\mathrm{m}}$ is the temperature at which the degradation rate is maximum. 


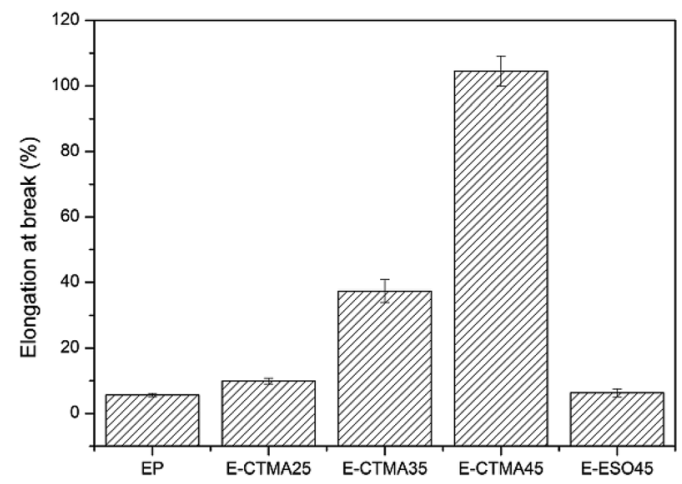

(a)

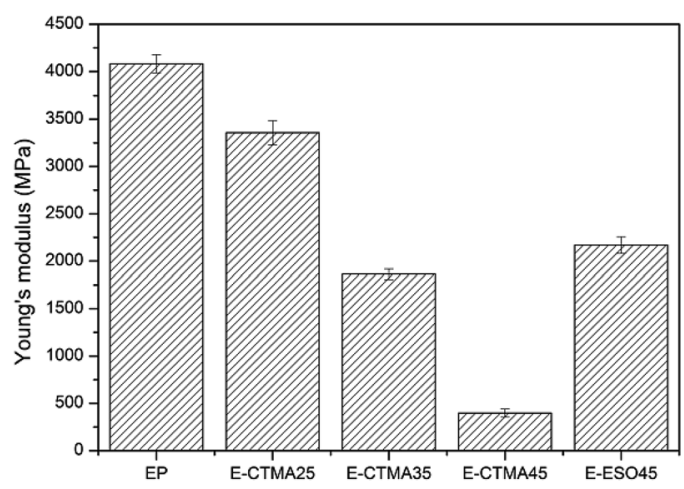

(c)

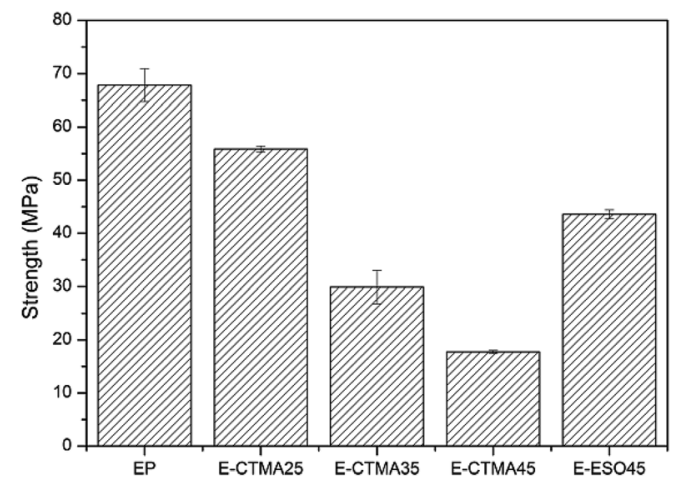

(b)

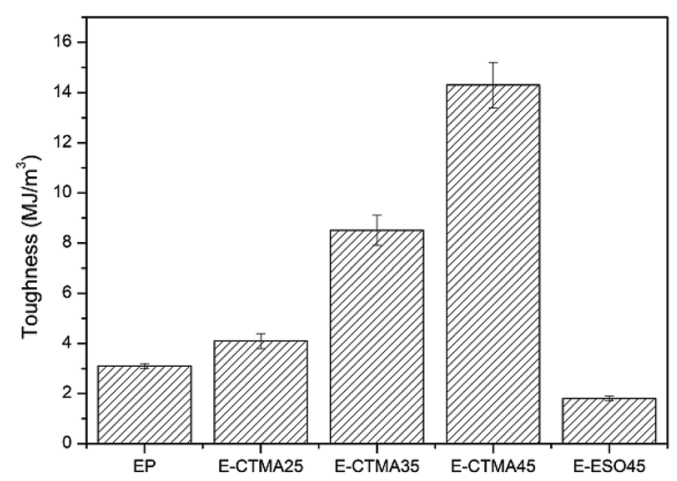

(d)

Fig. 11 Mechanical properties of the neat epoxy resin and E-CTMA thermosets.

commercial ESO is about $260 \mathrm{~g} \mathrm{~mol}^{-1}$. In other words, each ESO molecule contains more than 3 epoxy groups on an average, which may react with the curing agent and avoid a sharp decrement in the cross-linking density. Therefore, the reduction of thermal stability is less. ${ }^{35}$

\section{Mechanical properties}

The mechanical properties of all thermosets are shown in Fig. 11. As can be seen, the elongation-at-break, which indicates the toughness of the material, has increased dramatically from $5.6 \%$ to $104.5 \%$; this suggests that CTMA can effectively toughen the epoxy curing system. This is owing to the flexibility of the modifier, which may restrict crack extension and induce crazes. ${ }^{40}$ Compared with other tung oil-based products synthesized by the Diels-Alder reaction, whose elongation-at-break is less than 5\%, E-CTMAs show higher toughness. ${ }^{21,23,24}$ However, an increase in the content of CTMA decreases the tensile strength and Young's modulus. Because the addition of CTMA reduces the cross-linking density and enhances ductility, the modified samples are less sensitive to deformation; this leads to lower Young's modulus. ${ }^{41,42}$ In addition, due to the presence of less covalent bonds, the samples are unable to withstand high stress; this accounts for the decrement of tensile strength. Furthermore, toughness can be indicated in terms of fracture energy. The increasing toughness, calculated by integrating the area under stress-strain curves, ${ }^{43}$ demonstrates that CTMA can effectively toughen the epoxy curing system as well.

The sample E-ESO45 was used for comparison. Obviously, although E-ESO45 has higher tensile strength and Young's modulus, its toughness value is much lower than that of ECTMA45; this is mainly attributed to its low elongation-atbreak. Moreover, many studies on the toughening of epoxy resin with ESO have been reported, which indicate that ESO shows limited improvement in the elongation-at-break..$^{20,35,38,39}$ Hence, in terms of elongation-at-break and toughness value, CTMA exhibits excellent toughening ability, which is better than ESO. Moreover, the mechanical properties suggest that the epoxy resin modified by CTMA is a potential material that can be used as a coating, pouring sealant and other casting material and has higher requirement for toughness but less demand for strength. ${ }^{44}$ However, owing to the higher viscosity of some formulations, some solvents should be used to reduce the viscosity for applications.

\section{Conclusions}

Epoxy resin modified by CTMA shows a great improvement in toughness when compared with the neat epoxy resin and the sample modified by ESO. By acting as a flexible cross-linker, CTMA reduces the cross-linking density of epoxy resin, simultaneously increasing the elongation-at-break and toughness 
value. However, the incorporation of flexible aliphatic chains decreases the tensile strength, Young's modulus and $T_{\mathrm{g}}$ value; this is similar to the results of rubber-modified epoxy resin. Due to the reaction between the carboxyl groups and the epoxy groups, no phase separation has been detected by thermal characterization and SEM. Based on these characteristics, the epoxy resin modified by CTMA is a potential material for application as a coating, pouring sealant and casting material; moreover, the results demonstrate that the modified resins obtained from renewable feedstock, such as tung oil, will be potential alternatives to petroleum-based modified resins in the future.

\section{Conflicts of interest}

There is no conflict of interest to declare.

\section{Acknowledgements}

This work was financially supported by the National "Thirteenth Five-Year" Key Research and Development Plan (2017YFD0601304) and the Science Foundation of Key Laboratory of Biomass Energy and Materials of Jiangsu Province (JSBEM-S-201703).

\section{References}

1 Y. Huang, Y. Tian, Y. Li, X. Tan, Q. Li, J. Cheng and J. Zhang, RSC Adv., 2017, 7, 49074-49082.

2 R. Pearson and A. Yee, J. Mater. Sci., 1989, 24, 2571-2580.

3 A. F. Yee and R. A. Pearson, J. Mater. Sci., 1986, 21, 24622474.

4 H. Ren, X. Zhan, Q. Zhang and F. Chen, RSC Adv., 2016, 6, 35621-35627.

5 H. Ren, X. Zhan, Q. Zhang and F. Chen, J. Appl. Polym. Sci., 2016, 133, 42826.

6 X. Fernández-Francos, D. Foix, À. Serra, J. M. Salla and X. Ramis, React. Funct. Polym., 2010, 70, 798-806.

7 M. Flores, X. Fernández-Francos, F. Ferrando, X. Ramis and À. Serra, Polymer, 2012, 53, 5232-5241.

8 X. Fei, W. Wei, Y. Tang, Y. Zhu, J. Luo, M. Chen and X. Liu, Eur. Polym. J., 2017, 90, 431-441.

9 D. Foix, A. Serra, L. Amparore and M. Sangermano, Polymer, 2012, 53, 3084-3088.

10 L. Calabrese and A. Valenza, Eur. Polym. J., 2003, 39, 13551363.

11 K. Srivastava, A. K. Rathore and D. Srivastava, Spectrochim. Acta, Part A, 2018, 188, 99-105.

12 J. Xi and Z. Yu, J. Appl. Polym. Sci., 2018, 135, 45767.

13 L. Hader-Kregl, G. M. Wallner, R. W. Lang and B. Strauß, J. Appl. Polym. Sci., 2018, 135, 46094.

14 J. Huang and X. Nie, Polym. Int., 2016, 65, 835-840.

15 R. J. Day, P. A. Lovell and A. A. Wazzan, Polym. Int., 2001, 50, 849-857.

16 G. M. Roudsari, A. K. Mohanty and M. Misra, ACS Sustainable Chem. Eng., 2017, 5, 9528-9541.
17 S. Miao, P. Wang, Z. Su and S. Zhang, Acta Biomater., 2014, 10, 1692-1704.

18 S. Qureshi, J. A. Manson, J. C. Michel, R. W. Hertzberg and L. H. Sperling, in Characterization of Highly Cross-linked Polymers, American Chemical Society, 1984, vol. 243, ch. 8, pp. 109-124.

19 H. Miyagawa, A. K. Mohanty, M. Misra and L. T. Drzal, Macromol. Mater. Eng., 2004, 289, 636-641.

20 D. Ratna, Polym. Int., 2015, 50, 179-184.

21 K. Huang, J. Xia, X. Yang, M. Li and H. Ding, Polym. J., 2010, 42, 51-57.

22 L. Hou and Y. Liu, J. Appl. Polym. Sci., 2012, 126, 1572-1579.

23 K. Huang, Z. Liu, J. Zhang, S. Li, M. Li, J. Xia and Y. Zhou, Biomacromolecules, 2014, 15, 837-843.

24 K. Huang, P. Zhang, J. Zhang, S. Li, M. Li, J. Xia and Y. Zhou, Green Chem., 2013, 15, 2466-2475.

$25 \mathrm{~J}$. M. Barton, The application of differential scanning calorimetry (DSC) to the study of epoxy resin curing reactions, 1985.

26 A. Saikia and N. Karak, Polym. Degrad. Stab., 2017, 135, 8-17.

27 A. R. Kannurpatti, J. W. Anseth and C. N. Bowman, Polymer, 1998, 39, 2507-2513.

28 L. Wang, Y. Liang, Q. Yu, S. Chen, J. Zhang, M. Miao and D. Zhang, Polym. Compos., 2018, 39, 2046-2055.

29 X. Miao, Y. Meng and X. Li, Polymer, 2015, 60, 88-95.

30 L. Lijuan, M. Yan, Q. Teng, L. Zhuoxin, Y. Jing, C. Xingzhong and L. Xiaoyu, Polym. Compos., 2013, 34, 1051-1060.

31 T. Li, H. Qin, Y. Liu, X. Zhong, Y. Yu and A. Serra, Polymer, 2012, 53, 5864-5872.

32 J.-P. Yang, Z.-K. Chen, G. Yang, S.-Y. Fu and L. Ye, Polymer, 2008, 49, 3168-3175.

33 M. Morell, M. Erber, X. Ramis, F. Ferrando, B. Voit and A. Serra, Eur. Polym. J., 2010, 46, 1498-1509.

34 L. Luo, T. Qiu, Y. Meng, L. Guo, J. Yang, Z. Li, X. Cao and X. Li, RSC Adv., 2013, 3, 14509-14520.

35 S. K. Sahoo, S. Mohanty and S. K. Nayak, RSC Adv., 2015, 5, 13674-13691.

36 P. Chand, C. V. Reddy, J. G. Verkade, T. Wang and D. Grewell, Energy Fuels, 2009, 23, 989-992.

37 C. Yan, L. Yang, L. Ma, D. E. Finlow, S. Lin and K. Song, J. Therm. Anal. Calorim., 2013, 113, 939-945.

38 S. Kumar, S. K. Samal, S. Mohanty and S. K. Nayak, Ind. Eng. Chem. Res., 2017, 56, 687-698.

39 S. K. Sahoo, S. Mohanty and S. K. Nayak, Chin. J. Polym. Sci., 2015, 33, 137-152.

40 J. M. Misasi, Q. Jin, K. M. Knauer, S. E. Morgan and J. S. Wiggins, Polymer, 2017, 117, 54-63.

41 H. Ren, X. Zhan, Q. Zhang, G. Zhang and F. Chen, Polymer, 2016, 92, 222-230.

42 H. Ren, X. Zhan, Q. Zhang and F. Chen, J. Appl. Polym. Sci., 2017, 134, 44849.

43 J. Song, C. Chen, S. Zhu, M. Zhu, J. Dai, U. Ray, Y. Li, Y. Kuang, Y. Li and N. Quispe, Nature, 2018, 554, 224-239. 44 L. Ying and S. Mao, J. Appl. Polym. Sci., 1996, 61, 2059-2063. 